\title{
Hypoxia-induced Rab11-family interacting protein 4 expression promotes migration and invasion of colon cancer and correlates with poor prognosis
}

\author{
JIAN-ZHANG WANG $^{1 *}$, SHOU-XING YANG ${ }^{1 *}$, FANGPENG YE $^{2}$, XUAN-PING XIA ${ }^{1}$, \\ XIAO-XIAO SHAO ${ }^{1}$, SHENG-LONG XIA ${ }^{1}$, BO ZHENG $^{1}$ and CHANG-LONG XU ${ }^{1}$ \\ ${ }^{1}$ Department of Gastroenterology, The Second Affiliated Hospital and \\ Yuying Children's Hospital of Wenzhou Medical University, Wenzhou, Zhejiang 325027; \\ ${ }^{2}$ Department of Gastroenterology, Ruian People's Hospital, Wenzhou, Zhejiang 325200, P.R. China
}

Received March 29, 2017; Accepted September 6, 2017

DOI: $10.3892 / \mathrm{mmr} .2017 .8283$

\begin{abstract}
Rab11-family interacting proteins (Rab11-FIPs) are associated with the progression of various tumors; however, their expression and clinical significance in colorectal cancer (CRC) remains largely undetermined. In this study, the clinical implications, functions and underlying mechanisms of Rab11-FIP4 in CRC were investigated. Immunohistochemical analysis revealed that expression of Rab11-FIP4 was significantly increased in human CRC tissues and correlated with poor prognosis of patients with CRC. Overexpression of Rab11-FIP4 in the CRC cell line significantly promoted cell proliferation, migration and invasion in vitro and tumor metastasis in vivo. Furthermore, the results of a co-immunoprecipitation assay and western blot analysis demonstrated that Rab11-FIP4 interacted with Rab11 and insulin-like growth factor 1 receptor, and increased the phosphorylation of extracellular signal-regulated kinase $1 / 2$ and AKT serine/threonine kinase. In addition, hypoxia contributed to the upregulation of Rab11-FIP4 expression via hypoxia-inducible factor-1 $\alpha$ activation of the Rab11-FIP4 promoter. In conclusion, the results of the present study suggest that Rab11-FIP4 may act as an oncogene in CRC, and may be a potential therapeutic target for the treatment of patients with CRC.
\end{abstract}

Correspondence to: $\mathrm{Dr}$ Chang-Long $\mathrm{Xu}$, Department of Gastroenterology, The Second Affiliated Hospital and Yuying Children's Hospital of Wenzhou Medical University, 109 West Xueyuan Road, Wenzhou, Zhejiang 325027, P.R. China

E-mail: xchlong@163.com

*Contributed equally

Key words: colorectal cancer, Rab11-family interacting proteins, hypoxia, insulin-like growth factor 1 receptor, metastasis

\section{Introduction}

Colorectal cancer (CRC) is one of the most common cancers worldwide $(1,2)$. In China, CRC is among the five most prevalent types of cancer, with 376,300 new diagnoses made in 2015 alone (3). Furthermore, CRC is one of the five leading causes of cancer-associated mortality in China, with 191,000 CRC-associated mortalities reported in 2015 (3). Despite recent improvements in diagnostic technologies and clinical treatments, the prognoses for patients with CRC remain poor, particularly in patients with advanced stage CRC, due to risks of relapse and metastasis (4). It has previously been estimated that $\sim 50 \%$ of patients with CRC develop distant metastases, particularly liver metastases (5). However, the exact molecular mechanisms underlying CRC progression and metastasis development remain undetermined.

Rab11 family interacting proteins (Rab11-FIPs), comprising of Rip11, Rab11-FIP1, Rab11-FIP2, Rab11-FIP3, Rab coupling protein (RCP) and Rab11-FIP4, have previously been revealed as regulators of multiple Rab and ADP-ribosylation factor GTPases (6-9). Rab11-FIP4 was first identified in a human cDNA project as a gene termed KIAA1821 (10). Rab11-FIP4 shares a common C-terminal Rab11 binding domain (RBD) with the other FIPs, and selectively interacts with the GTP-bound form of Rab11 (11). Furthermore, Rab11-FIP4 belongs to the class II FIPs, and its N-terminus contains an EF-hand calcium-binding motif (12). In addition, Rab11-FIP4 localizes to the endosomal recycling compartment and functions as a downstream modulator of Rab11 to regulate vesicle trafficking $(8,13)$. A previous study demonstrated that Rab11-FIP4 interacts with Rab11 in a GTP-dependent manner, and that overexpression of Rab11-FIP4 condenses the Rab11 positive compartment in HeLa cells (14). In addition, previous studies have also revealed that Rab11-FIP4 is involved in cell proliferation and differentiation during retinal development, also in a Rab11-independent manner $(15,16)$. Furthermore, a recent study demonstrated that Rab11-FIP4 has a pro-metastatic function via activation of the mechanistic target of rapamycin/AKT1 substrate 1 pathway in hepatocellular carcinoma (HCC) (17). However, the potential implication 
of Rab11-FIP4 activity in CRC remains undetermined. In the present study, the clinical implications, functions and underlying mechanisms of Rab11-FIP4 in CRC were investigated. The results of the present study demonstrated that Rab11-FIP4 could be a promising therapeutic target for CRC treatment.

\section{Materials and methods}

Patients and sample collection. CRC samples and their corresponding non-tumorous (NT) samples (50 pairs of tissue samples and 100 CRC tissues) were obtained at The Second Affiliated Hospital and Yuying Children's Hospital of Wenzhou Medical University (Wenzhou, China) between January 2009 and October 2015. Pathological diagnosis of CRC was confirmed by a pathologist without knowledge of patient characteristics. All patients included in this study provided signed consent, and the study was approved by the Ethics Committee of Wenzhou Medical University.

Immunohistochemical staining. Tissue microarrays (TMAs) were constructed, and immunohistochemistry (IHC) staining and immunostaining scoring were performed according to previous studies (18). Briefly, TMAs were dewaxed, rehydrated, and blocked for 90 min with $2 \%$ normal goat serum at $37^{\circ} \mathrm{C}$ (Gibco; Thermo Fisher Scientific, Inc., Waltham, MA, USA). Vectastain Elite ABC Kit (Vector Laboratories, Inc., Burlingame, CA, USA) was used in IHC analysis. Tissues sections $(5 \mu \mathrm{m})$ were incubated with primary antibodies against Rab11FIP4 (cat. no. HPA021595; 1:50; Sigma-Aldrich; Merck KGaA, Darmstadt, Germany) and hypoxia-inducible factor 1- $\alpha$ (cat. no. MA1-16504; 1:100; Thermo Fisher Scientific, Inc.) overnight at $4^{\circ} \mathrm{C}$ in a humidified chamber. Horseradish peroxidase-conjugated secondary antibodies against rabbit and mouse immunoglobulins were developed for $20 \mathrm{~min}$ at $37^{\circ} \mathrm{C}$ with the DAB peroxidase substrate kit (cat. no. K500711-2; 1:1,000; Dako; Agilent Technologies, Inc., Santa Clara, CA, USA). Cell nuclei were stained with hematoxylin and eosin (cat. no. 51275; Sigma-Aldrich; Merck KGaA) for $90 \mathrm{sec}$ at $37^{\circ} \mathrm{C}$. Incubations with pre-immune serum were used as negative controls. Tissue sections were viewed with a microscope (x400 magnification; Nikon Eclipse 50i; Nikon Corporation, Tokyo, Japan). IOD was calculated using Image-Pro Plus software (version 6; Media Cybernetics, Inc., Rockville, MD, USA) and the mean IOD was calculated from three images per specimen.

Cell culture and hypoxic conditions. Human colorectal cancer cell lines (LoVo and HCT116) were obtained from the Shanghai Cell Bank, Chinese Academy of Sciences (Shanghai, China). LoVo and HCT116 cells were cultured in Dulbecco's Modified Eagle's Medium (DMEM; Gibco; Thermo Fisher Scientific, Inc.), which was supplemented with $10 \%$ fetal bovine serum (FBS; Gibco; Thermo Fisher Scientific, Inc.), penicillin (10 units/ml; Thermo Fisher Scientific Inc.) and streptomycin (10 mg/ml; GE Healthcare Life Sciences, Logan, UT, USA). Both cell lines were incubated in a humidified atmosphere at $37^{\circ} \mathrm{C}$. For hypoxic culture, cells were placed in a hypoxia incubator for $48 \mathrm{~h}$ in an atmosphere consisting of $94 \% \mathrm{~N}_{2}, 5 \% \mathrm{CO}_{2}$ and $1 \% \mathrm{O}_{2}$.
Construction of Rab11-FIP4 overexpression lentivirus. The Rab11-FIP4 opening reading frame sequence (NM_032932.5) was cloned into the pWPXL vector (Addgene, Inc., Cambridge, MA, USA), and the pWPXL-Rab11FIP4 recombinant plasmid was constructed. Plasmids were purchased from Addgene, Inc. (Cambridge, MA, USA). Using Lipofectamine $2000^{\mathrm{TM}}$ (Thermo Fisher Scientific, Inc.), 293T cells were co-transfected with pWPXL-Rab11FIP4, psPAX2 (the packaging plasmid) and pMD2.G (the envelope plasmid). Following this, the lentivirus was harvested $48 \mathrm{~h}$ post-transfection, and CRC cells were infected with the lentivirus $(\mathrm{MOI}=10)$ in the presence of polybrene $(6 \mu \mathrm{g} / \mathrm{ml}$; Sigma-Aldrich; Merck KGaA, Darmstadt, Germany).

Reverse transcription-quantitative polymerase chain reaction (RT-qPCR). Total RNA was extracted from cells and tissues using TRIzol reagent (Thermo Fisher Scientific, Inc.), and RT was performed using PrimeScript ${ }^{\mathrm{TM}}$ RT Reagent Kit (Takara Biotechnology Co., Ltd., Dalian, China) in a total volume of $50 \mu \mathrm{l}$. The $\mathrm{RT}$ reaction proceeded for $15 \mathrm{~min}$ at $37^{\circ} \mathrm{C}$ followed by $5 \mathrm{sec}$ at $85^{\circ} \mathrm{C}$, according to manufacturer's instructions. The cDNA was stored at $-20^{\circ} \mathrm{C}$. SYBR Premix Ex Taq (Takara Biotechnology Co., Ltd.) was subsequently used to perform qPCR. The PCR conditions were as follows: $95^{\circ} \mathrm{C}$ for $15 \mathrm{sec}$ followed by 35 cycles of $95^{\circ} \mathrm{C}$ for $5 \mathrm{sec}$ and $60^{\circ} \mathrm{C}$ for $40 \mathrm{sec} . \beta$-actin was used as loading control, and the relative expression levels were determined by the following equation: $2^{-\Delta \Delta \mathrm{Cq}}\left(\Delta \mathrm{Cq}=\Delta \mathrm{Cq}^{\text {target }}-\Delta \mathrm{Cq}^{\beta \text {-actin }}\right)(19)$. The primers used in this study were as follows: Rab11FIP4 forward, 5'-CTGCTC TCAATGCTGCAAGA-3' and reverse, 5'-TCGCAAGAGTCA ATGCTGTC'; insulin-like growth factor 1 receptor (IGF1R) forward, 5'-TCGACATCCGCAACGACTATC-3' and reverse, 5'-CCAGGGCGTAGTTGTAGAAGAG-3'; $\beta$-actin forward, 5'-TTGTTACAGGAAGTCCCTTGCC-3' and reverse, 5'-ATG CTATCACCTCCCCTGTGTG-3'.

Western blot. Western blot was performed according to a previous study (18). Briefly, tumor cells and tissues were lysed, and cell debris was removed. Lysates of cells or tissues were prepared with T-PER tissue protein extraction reagent (Pierce; Thermo Fisher Scientific, Inc.) supplemented with proteinase inhibitors (Roche Applied Science, Switzerland) and phosphatase inhibitors (Roche Diagnostics, Basel, Switzerland). To investigate the effects of IGF1R inhibition on protein expression, HCT116-Rab11-FIP4 cells were treated with NVP-AEW541 (5 $\mu \mathrm{M})$ for $12 \mathrm{~h}$ before they were lysed. Protein concentrations were determined with a bicinchoninic assay kit (Pierce; Thermo Fisher Scientific, Inc.). A total of 60-80 $\mu \mathrm{g}$ protein/sample was separated on 10\% SDS-PAGE and immobilized to nitrocellulose membranes (Bio-Rad Laboratories Inc., Hercules, CA, USA). The nitrocellulose membranes were then blocked for $\sim 2 \mathrm{~h}$ with $5 \%$ fat-free milk at room temperature. Specific primary antibodies against Rab11-FIP4 (cat. no. sc-165331; 1:1,000; Santa Cruz Biotechnology Inc., Dallas, TX, USA), Rab11 (cat. no. sc-6565; 1:1,000; Santa Cruz Biotechnology Inc.), hypoxia-inducible factor-1 $\alpha$ (HIF-1 $\alpha$; cat. no. sc-10790; 1:1,000; Santa Cruz Biotechnology Inc.), IGF1R (cat. no. sc-7952; 1:1,000; Santa Cruz Biotechnology Inc.), phosphorylated (p)-extracellular signal-regulated kinase 1/2 (ERK1/2; cat. no. sc-101760; 
1:1,000; Santa Cruz Biotechnology Inc.), total-ERK1/2 (cat. no. sc-514302; 1:1,500; Santa Cruz Biotechnology Inc.), p-AKT serine/threonine kinase (AKT; cat. no. sc-7985-R; 1:1,000; Santa Cruz Biotechnology Inc.), total-AKT (cat. no. sc-8312; 1:1,500; Santa Cruz Biotechnology, Inc.), and $\beta$-actin (cat. no. sc-58673; 1:3,000; Santa Cruz Biotechnology, Inc.) were incubated at $4{ }^{\circ} \mathrm{C}$ overnight. Following incubation with horseradish peroxidase-conjugated secondary anti-rabbit or anti-mouse antibodies (BS12478 or BS13278; 1:5,000; Bioworld Technology, Inc., St. Louis Park, MN, USA) for $1 \mathrm{~h}$ at room temperature. Bands were visualized using an enhanced chemiluminescence reagent (ECL Plus; GE Healthcare, Chicago, IL, USA). ImageLab software (version 4.1; Bio-Rad Laboratories, Inc.) was used for image acquisition and densitometric analysis of bands.

Co-immunoprecipitation (Co-IP). Pierce ${ }^{\circledR}$ Co-IP kit (Thermo Fisher Scientific, Inc.) was used to perform a Co-IP assay according to the manufacturer's instructions. In brief, an antibody against Rab11-FIP4 (cat. no. NBP2-45496; 1:50; Novus Biologicals, LLC, Littleton, CO, USA) was immobilized to AminoLink Plus Coupling Resin (Thermo Fisher Scientific, Inc.) at room temperature. Control resin was used to prevent nonspecific binding and following this, cell lysates were added to the spin column, which contained the immobilized antibody resin, and incubated overnight at $4^{\circ} \mathrm{C}$. Following this, gentle elution buffer was used to disassociate the interacting proteins from the immobilized antibodies.

Dual-luciferase reporter assay. Dual-luciferase reporter assay (Promega Corporation, Madison, WI, USA) was then used to detect luciferase activity. HCT116 cells were plated in a 96-well plate. Using Lipofectamine $2000^{\mathrm{TM}}$ (Thermo Fisher Scientific, Inc.), pGL3 (Firefly luciferase) constructs of the truncated Rab11-FIP4 promoter were co-transfected with pCMV-HIF1 $\alpha$ (200 ng/well) or pCMV-vector (200 ng/well) and pRL-TK (6 ng/well; Renilla luciferase) in HCT116 cells. Empty plasmid of pGL3 was defined as pGL3-Basic. The pGL3 and pRL-TK vectors were purchased from Promega Corporation (Madison, WI, USA). The Pcmv vector was purchased from Addgene, Inc. (Cambridge, MA, USA). The plate was washed with PBS $6 \mathrm{~h}$ post-transfection and replaced with fresh culture medium. The firefly and Renilla luciferase activity was then measured $48 \mathrm{~h}$ post-transfection using the Dual-Luciferase ${ }^{\circledR}$ Reporter Assay System (Promega Corporation). Firefly luciferase activity was normalized to the Renilla luciferase activity.

Cell proliferation assay. The proliferation of CRC cells was detected using the Cell Counting Kit-8 (CCK-8) reagent (Dojindo Molecular Technologies, Inc., Kumamoto, Japan). Briefly, cells $\left(4 \times 10^{3}\right.$ per well) were seeded in 96-well culture plates and then cultured at $37^{\circ} \mathrm{C}$ for $0,24,48$ and $72 \mathrm{~h}$ after attachment. CCK-8 assay buffer (100 $\mu \mathrm{l} /$ per well) was then added into the plates, and $450 \mathrm{~nm}$ optical density values were obtained using a microplate reader (BioTek Instruments, Inc., Winooski, VT, USA) according to manufacturer's protocol.

Plate colony formation assay. LoVo and HCT116 cells (800 per well) were seeded into separate 6-well plates for 8 days. Culture medium was changed every 2 days. Following this, cells were washed with PBS, fixed with $4 \%$ paraformaldehyde for $15 \mathrm{~min}$ at $37^{\circ} \mathrm{C}$ and stained with $1 \%$ crystal violet solution (Merck KGaA) for 5 min. Colonies with >50 cells were counted using a light microscope (x400 magnification).

Transwell (migration) and Matrigel (invasion) assays. Transwell chambers ( $8 \mu \mathrm{m}$; BD Biosciences, Franklin Lakes, NJ, USA) were placed in 24-well plates. In order to perform the migration assay, CRC cells $\left(2 \times 10^{4}\right)$ in serum free DMEM were seeded on the upper chamber of Transwell chambers and medium supplemented with $15 \%$ FBS was then added to the lower chamber. Following incubation for $20 \mathrm{~h}$, migrated CRC cells were stained for 5 min with $1 \%$ crystal violet and imaged using a CKX41 light microscope (Olympus Corporation, Tokyo, Japan) at x200 magnification. The mean number of migrated cells was determined by counting the number of cells in three random fields from three replicate wells. In order to carry out the invasion assay, Transwell chambers were coated with Matrigel (BD Biosciences). CRC cells $\left(4 \times 10^{4}\right)$ in serum-free DMEM were seeded on the upper chamber of Transwell chambers, and medium supplemented with $15 \%$ FBS was then added to the lower chamber. Following incubation for $36 \mathrm{~h}$, migrated cells were then stained, imaged and counted as detailed in the aforementioned migration assay. Images of three random fields from three replicate wells were obtained, and the numbers of migrated or invasion cells were then counted. To investigate the potential effects of ERK and AKT on migration and invasion, wHCT116-Rab11-FIP4 cells were pretreated with either ERK inhibitor (U0126; $5 \mu \mathrm{M}$ ) or AKT inhibitor (LY294002; $10 \mu \mathrm{M}$ ) for $6 \mathrm{~h}$, respectively, before seeding onto the upper chamber of Transwell chambers.

Human phospho-kinase array. HCT116-vector or HCT116-Rab11FIP4 cells were used in the phosphokinase array assays, according to the manufacturer's instructions (Proteome Profiler; cat. no. ARY003B; R\&D Systems, Inc., Minneapolis, MN, USA). Briefly, cell lysates $(800 \mu \mathrm{g})$ were mixed with array buffer and incubated with pre-blocked array membrane at $4{ }^{\circ} \mathrm{C}$ overnight. Membranes were then washed and incubated with the primary antibody cocktail for $2 \mathrm{~h}$, followed by washing and incubation with the secondary antibody for $30 \mathrm{~min}$ at $37^{\circ} \mathrm{C}$. Membranes were washed again and subjected to chemiluminescent detection.

In vivo metastasis assay. The Medical Experimental Animal Care Commission, Wenzhou Medical University, approved all animal experimentation. Male BALB/c nude mice (age, 6 weeks; weight, 15-18 g) were purchased from Shanghai Laboratory Animal Center (Shanghai, China) and randomly divided into two groups ( 8 mice per group). A total of 4x10 ${ }^{6}$ HCT116 cells $/ 200 \mu \mathrm{l}$ PBS were injected into the tail veins of nude mice. All mice were maintained at $25^{\circ} \mathrm{C}$ and $\sim 60 \%$ humidity with a $12 \mathrm{~h}$ light/dark cycle with free access to food and water. After 7 weeks the mice were sacrificed, and their lung tissues were dissected and fixed with $10 \%$ formalin at room temperature for $48 \mathrm{~h}$. Tumor sections $(5 \mu \mathrm{m})$ were subsequently incubated with Mayer's hematoxylin (Sigma-Aldrich; Merck KGaA) for $1 \mathrm{~min}$ at room temperature and washed with running tap water for $3 \mathrm{~min}$. The tissues were immerged within Eosin Y solution (Sigma-Aldrich; Merck KGaA) for $20 \mathrm{sec}$ at room temperature. 
A

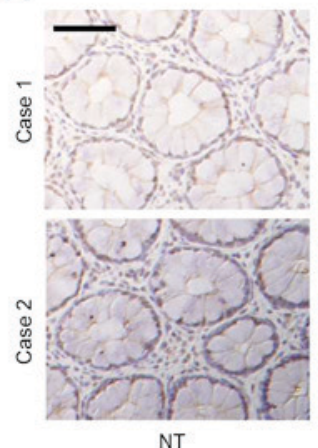

NT

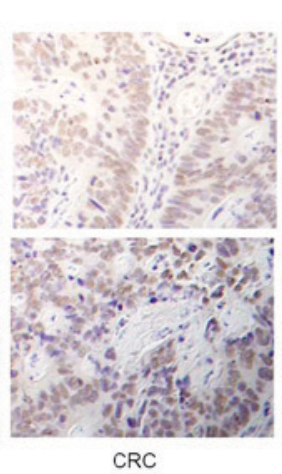

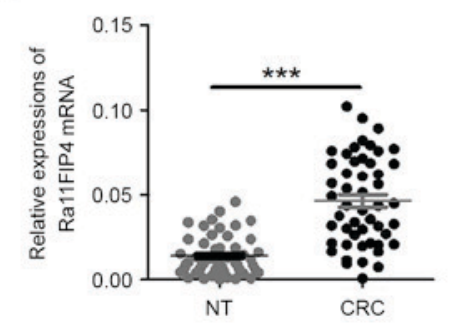

B

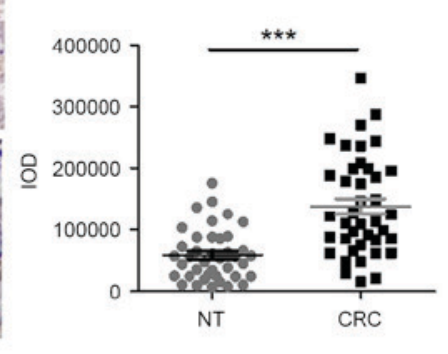

E

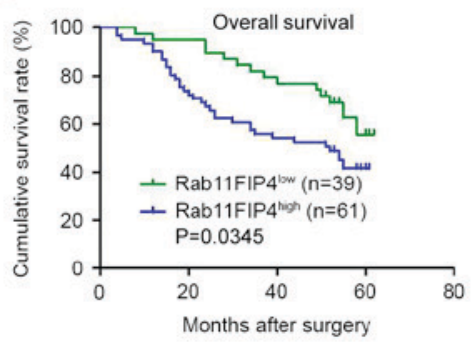

C

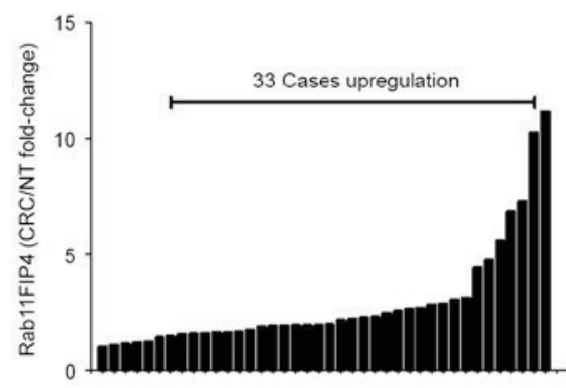

$\mathrm{F}$

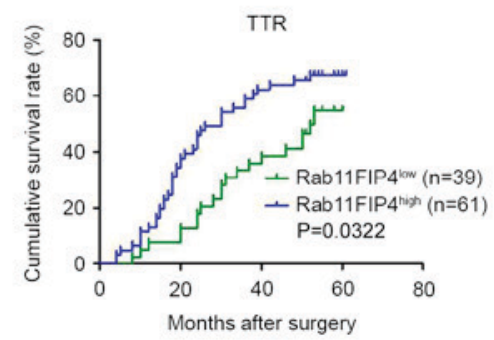

Figure 1. High levels of Rab11-FIP4 predicts poor prognosis of patients with CRC. Expression of Rab11-FIP4 in was determined in 40 pairs of CRC tissues and corresponding NT tissues detected by IHC analyses. (A) Representative IHC images of Rab11FIP4 expression in CRC tissues and corresponding NT (magnification, x400; scale bar, $100 \mu \mathrm{m}$ ). (B) IOD for Rab11-FIP4 analyzed from 40 paired samples of patients with CRC. (C) Fold change of the Rab11-FIP4 IOD values in paired CRC samples $(n=40)$. (D) mRNA levels of Rab11-FIP4 in 40 pairs of CRC tissues and corresponding NT tissues detected by reverse transcription-quantitative polymerase chain reaction. $\beta$-actin was used as loading control. Kaplan-Meier analysis of (E) overall survival and (F) TTR were performed according to the mRNA levels of Rab11-FIP4 in 100 cases of CRC in tissue microarrays detected by IHC with patients divided into Rab11-FAPlow and Rab11-FIP high groups. ${ }^{* * *} \mathrm{P}<0.001$. IHC, immunohistochemistry; NT, non-tumoral; CRC, colorectal cancer; IOD, integrated optical density; Rab11-FIP4, Rab11-family interacting protein 4; TTR, time to recurrence.

Statistical analysis. GraphPad Prism 5.0 software (La Jolla, CA, USA) was used to analyze all data in the present study. Data were presented as the mean \pm standard deviation. Multiple group comparisons were performed with one-way analysis of variance, followed by Tukey's test. Overall survival was determined by the Kaplan-Meier method and compared with the log-rank test. $\mathrm{P}<0.05$ was considered to indicate a statistically significant difference.

\section{Results}

Rab11-FIP4 expression is upregulated in CRC tissues and high expression of Rabl1-FIP4 is associated with poor prognosis of patients with CRC. Using IHC analyses, the expression patterns of Rab11-FIP4 in CRC tissues and corresponding NT tissues in CRC samples were investigated. As presented in Fig. 1A, the majority of Rab11-FIP4 was distributed in the cytoplasm and membranes of CRC cells in clinical samples. The expression level of Rab11-FIP4 was significantly increased in CRC tissues compared with paired NT tissues in 33 of the 40 patients with CRC (Fig. 1B and C). Similar results were revealed by the RT-qPCR analyses (Fig. 1D). Furthermore, the clinical significance of Rab11-FIP4 expression was investigated in patients with CRC. The protein level of Rab11-FIP4 in 100 patients with CRC was detected via TMAs using IHC analyses. Subsequently, patients with CRC were divided into two groups based on their respective IHC scores: High Rab11-FIP4 expression group $(n=61)$ and low Rab11-FIP4 expression group $(n=39)$. The clinical and pathological characteristics of patients with CRC included in the present study are presented in Table I. Kaplan-Meier survival analyses revealed that the high Rab11-FIP4 group $(n=61)$ demonstrated a reduced overall survival time period $(\mathrm{P}<0.05$; Fig. 1E) and a higher tendency for CRC recurrence $(\mathrm{P}<0.05$; Fig. 1F) compared with the low Rab11-FIP4 group $(n=39)$. These results suggest that Rab11-FIP4 may act as an oncogene in CRC progression, and that Rab11-FIP4 expression may be used as a biomarker for the prognostic prediction of patients with CRC.

Overexpression of Rabl1-FIP4 promotes the proliferation, migration and invasion of CRC cells in vitro, and tumor metastasis in vivo. In order to investigate the function of Rab11-FIP4 in CRC cells, two common CRC cell lines, HCT116 and LoVo, were transfected with a lentivirus that stably expresses Rab11-FIP4. The results of the RT-qPCR and western blot analyses demonstrated that mRNA and protein levels of Rab11-FIP4 were significantly overexpressed in both HCT116 and LoVo cells transfected with the lentivirus (Fig. 2A and B). The CCK-8 assay revealed that overexpression of Rab11-FIP4 promoted the proliferation of CRC cells (Fig. 2C). Furthermore, the clonogenic assay demonstrated that overexpression of Rab11-FIP4 enhanced colony formation of CRC cells (Fig. 2D). In addition, Rab11-FIP4 overexpression significantly increased the migration and invasion of HCT116 and LoVo cells in vitro (Fig. 2E and F). Furthermore, the effect of Rab11-FIP4 expression on CRC metastasis was investigated in vivo. 
Table I. Patient characteristics.

\begin{tabular}{|c|c|c|c|}
\hline Variables & $\begin{array}{c}\text { No. of cases } \\
(n=100 \text { tumor tissues })^{a}\end{array}$ & $\begin{array}{c}\text { No. of cases } \\
(n=40 \text { tumor pairs })^{b}\end{array}$ & $\begin{array}{c}\text { No. of cases } \\
(n=50 \text { tumor pairs })^{c}\end{array}$ \\
\hline \multicolumn{4}{|l|}{ Sex } \\
\hline Male & 53 & 20 & 27 \\
\hline Female & 47 & 20 & 23 \\
\hline \multicolumn{4}{|l|}{ Age } \\
\hline$<60$ & 10 & 15 & 15 \\
\hline $60-69$ & 36 & 10 & 20 \\
\hline $70-79$ & 37 & 13 & 13 \\
\hline$\geq 80$ & 17 & 2 & 2 \\
\hline \multicolumn{4}{|l|}{ TNM } \\
\hline I-II & 52 & 14 & 21 \\
\hline III-IV & 48 & 26 & 29 \\
\hline \multicolumn{4}{|l|}{ Venous invasion } \\
\hline Positive & 55 & 32 & 41 \\
\hline Negative & 45 & 8 & 9 \\
\hline \multicolumn{4}{|l|}{ Tumor size } \\
\hline$\leq 5 \mathrm{~cm}$ & 43 & 15 & 18 \\
\hline$>5 \mathrm{~cm}$ & 57 & 25 & 32 \\
\hline \multicolumn{4}{|l|}{ Location } \\
\hline Right colon & 40 & 20 & 25 \\
\hline Left colon & 30 & 12 & 15 \\
\hline Rectum & 30 & 8 & 10 \\
\hline \multicolumn{4}{|l|}{ Histological type } \\
\hline Adenocarcinoma & 82 & 33 & 38 \\
\hline Non-adenocarcinoma & 18 & 7 & 12 \\
\hline \multicolumn{4}{|l|}{ Tumor differentiation } \\
\hline Well & 4 & 1 & 2 \\
\hline Moderate & 75 & 34 & 42 \\
\hline Poor & 21 & 5 & 6 \\
\hline
\end{tabular}

${ }^{\mathrm{a}}$ used for IHC; ${ }^{\mathrm{b}}$ used for IHC; ${ }^{\mathrm{u}}$ used for reverse transcription-quantitative polymerase chain reaction. TNM, tumor-node-metastasis stage; IHC, immunohistochemistry.

HCT116 cells transfected with either the Rab11-FIP4 or control vector were injected into nude mice via the lateral tail vein. At 8 weeks post-treatment, increased incidences of lung metastases and metastatic lesions were observed in the Rab11-FIP4 overexpression group of mice compared with the empty vector group (Fig. 2G-I).

Overexpression of Rab11-FIP4 increases the phosphorylation of ERK1/2 and AKT, which is mediated by IGF1R. IGF1R is frequently overexpressed in CRC, and promotes signaling pathways that regulate various functions, such as cell proliferation, tumor cell motility, invasion and metastasis. In the present study, it was revealed that Rab11-FIP4 could interact with IGF1R and regulate the expression level of IGF1R. Firstly, the effect of Rab11-FIP4 overexpression on the protein level of IGF1R was investigated using western blot analysis. The results demonstrated that the protein level of IGF1R was upregulated following Rab11-FIP4 overexpression (Fig. 3A).
However, it was revealed that overexpression of Rab11-FIP4 in HCT116 cells did not result in a corresponding increase in the level of IGF1R mRNA (Fig. 3B). These results suggest that Rab11-FIP4 may either protect the IGF1R protein from degradation, or promote IGF1R recycling. To further investigate this, a Co-IP assay was performed using HCT116-Rab11-FIP4 cells and corresponding control cells. The results suggested that Rab11-FIP4 forms a complex with Rab11 and IGF1R, and increased expression of Rab11-FIP4 increases the formation of this complex in HCT116 cells (Fig. 3C). In order to further investigate the signaling affected by IGF1R in CRC cells with increased expression of Rab11-FIP4, human phosphokinase array assays were performed. The results revealed that the phosphorylation levels of ERK1/2 and AKT were increased following overexpression Rab11FIP4 in HCT116 cells (Fig. 3D; fold change $\geq 2.0$ ). To determine whether these signaling molecules are activated by IGF1R in cells with increased Rab11-FIP4 expression, an IGF1R inhibitor was used to 
A

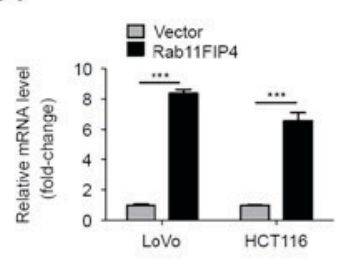

B

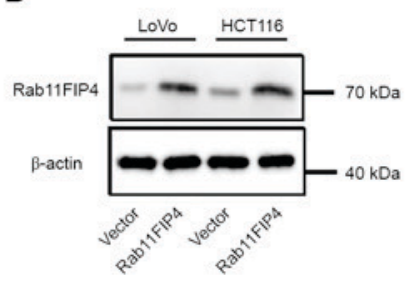

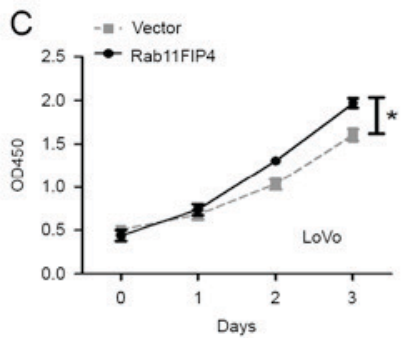

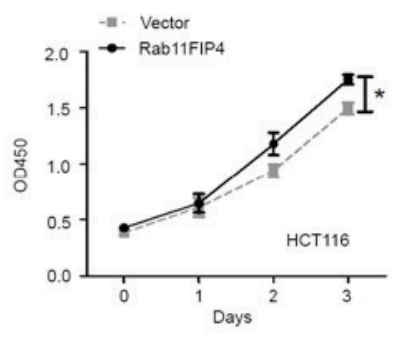

D
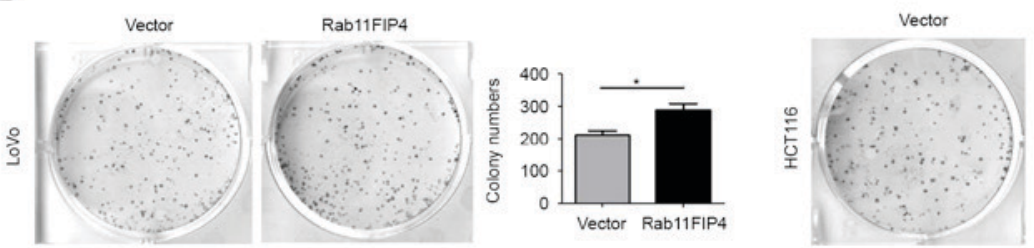

Rab11FIP4
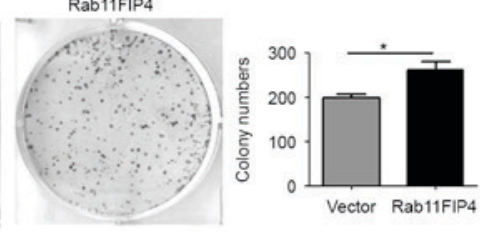

$\mathrm{E}$
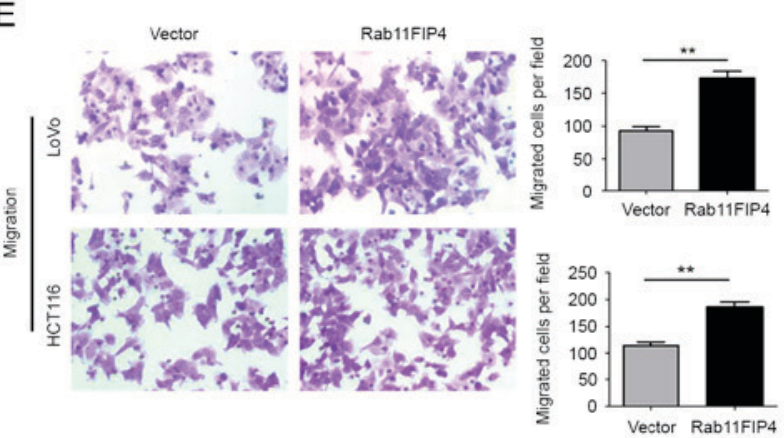

$\mathrm{F}$
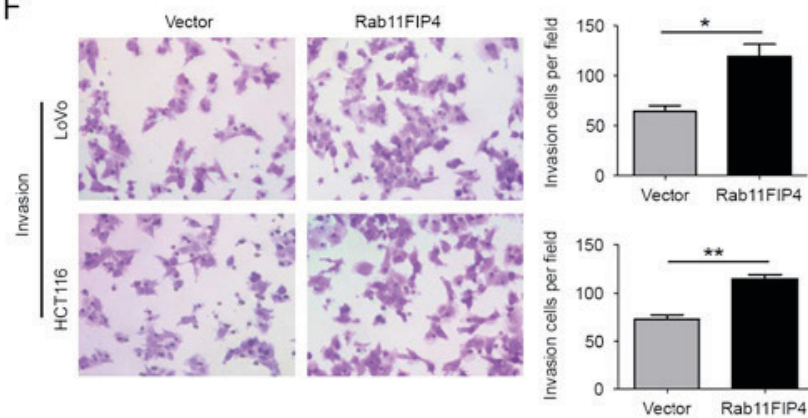

G

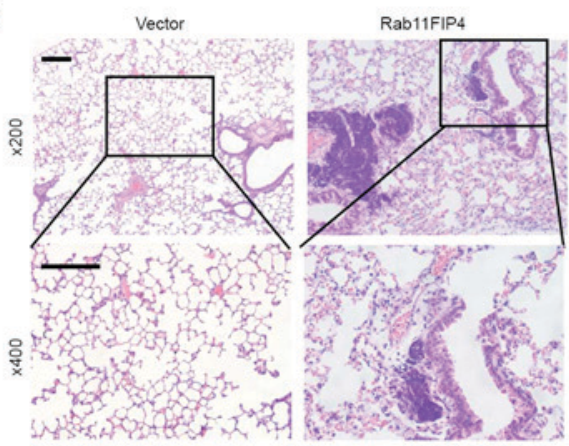

$\mathrm{H}$

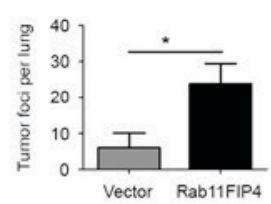

।

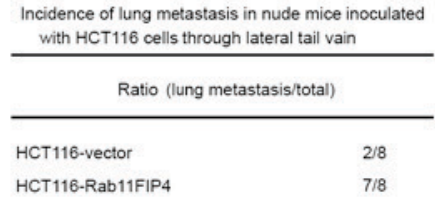

Figure 2. Overexpression of Rab11-FIP4 promotes proliferation, migration and invasion of CRC cells in vitro, and metastasis in vivo. HCT116 and LoVo cells with stable overexpression of Rab11-FIP4 were created using a lentiviral system. The mRNA level of Rab11-FIP4 was detected by (A) reverse transcription-quantitative polymerase chain reaction analysis and (B) western blot analysis. (C) Proliferation of CRC cells detected by Cell Counting kit 8 assays and (D) clonogenic assays revealed that increased expression of Rab11-FIP4 enhanced the colony formation capacity of CRC cells. (E) Migration and (F) invasion capabilities of CRC cells were detected using Transwell assays. (G) Representative hematoxylin and eosin staining images of mouse lung tissue sections from either HCT116-Vector or HCT116-Rab11-FIP4 groups (magnification, x200 and x400; scale bars=100 $\mu$ m). (H) Number of lung metastatic foci calculated microscopically. (I) Incidence of lung metastasis in nude mice inoculated with HCT116-Vector or HCT116-Rab11-FIP4 cells via the lateral tail vain $(\mathrm{n}=8) .{ }^{*} \mathrm{P}<0.05$; ${ }^{* *} \mathrm{P}<0.01$. CRC, colorectal cancer; Rab11-FIP4, Rab11-family interacting protein 4; OD, optical density.

treat HCT116 cells. The results demonstrated that p-ERK1/2 and $\mathrm{p}$-AKT levels were reduced following IGF1R inhibitor treatment (Fig. 3E). To further confirm the involvement of ERK1/2 and AKT signaling in the Rab11-FIP4-mediated process of CRC metastasis, HCT116 cells stably infected with Rab11-FIP4 were treated with inhibitors of ERK1/2 and AKT, respectively. The results revealed that CRC cells treated with ERK1/2 and AKT inhibitors had significantly reduced migration and invasion rates compared with the untreated cells (Fig. 3F and G). These results suggest that Rab11-FIP4 promotes CRC migration and invasion via the phosphorylation of ERK1/2 and AKT, which is regulated by IGF1R.
Expression of Rabl1-FIP4 is regulated by HIF-1 $\alpha$ in CRC cells. To investigate whether Rab11FIP4 is transcriptionally regulated by HIF- $1 \alpha$ in CRC, the expression levels of Rab11-FIP4 and HIF-1 $\alpha$ in six pairs of CRC tissues and their corresponding non-tumoral tissues were analyzed. The results demonstrated that the expression levels of Ratb11-FIP4 and HIF- $1 \alpha$ increased in CRC tissues compared with corresponding NT tissues (Fig. 4A). Furthermore, tissues with higher levels of HIF-1 $\alpha$ expression exhibited relatively higher levels of Rab11FIP4 (Fig. 4A). In addition to using IHC analysis to investigate Rab11-FIP4 expression, the expression of HIF- $1 \alpha$ in the same TMA containing 100 cases of CRC 
A

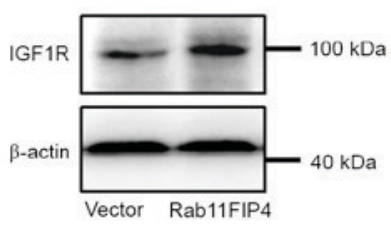

D

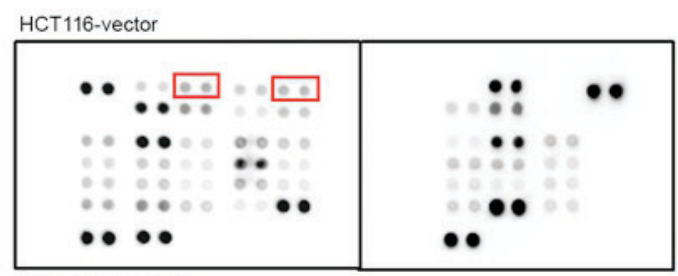

HCT116-Rab11FIP4

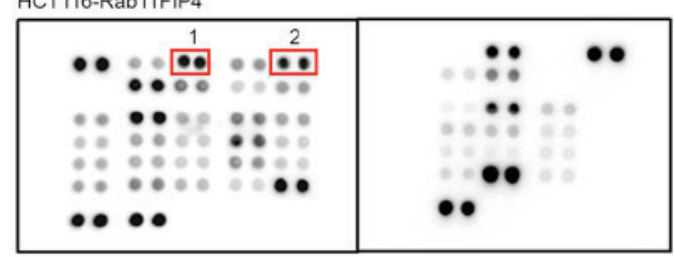

F

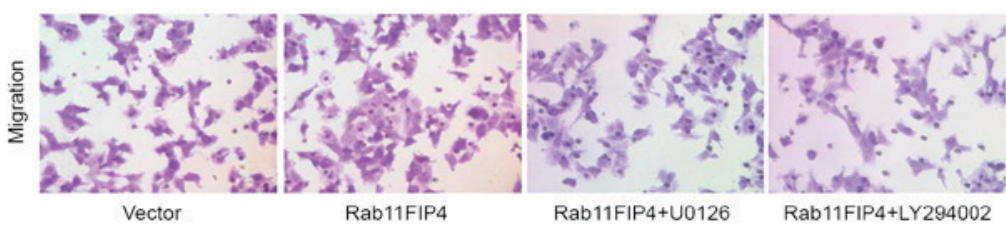

G

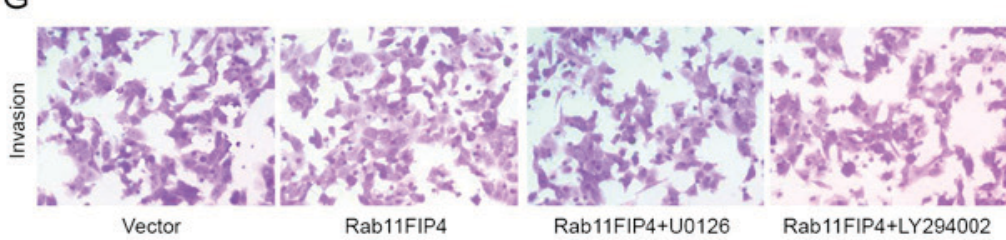

C

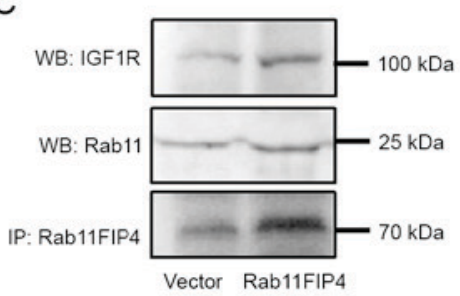

E

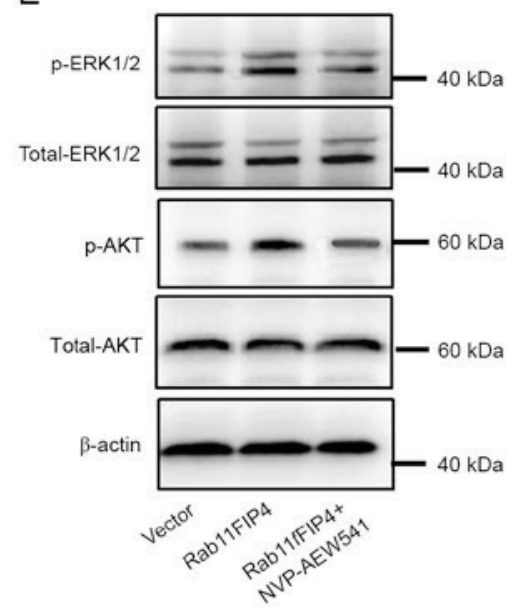

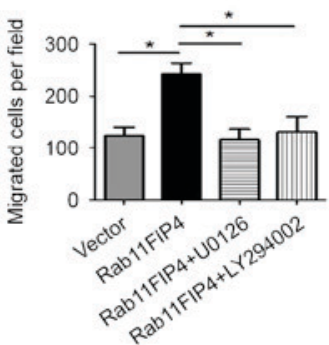

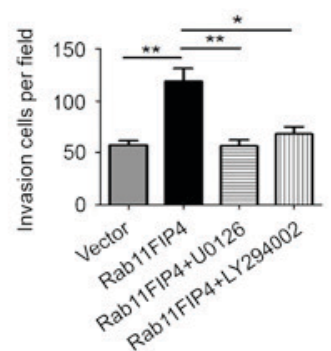

Figure 3. Overexpression of Rab11-FIP4 promotes migration and invasion of CRC cells via the phosphorylation of ERK1/2 and AKT, which is mediated by IGF1R. Expression level of IGF1R detected by (A) western blot analysis and (B) reverse transcription-quantitative polymerase chain reaction analysis. (C) Western blot analysis of protein interactions and lysate proteins resulting from Co-IP analysis of Rab11-FIP4, Rab11 and IGF1R. (D) Phosphokinase assay demonstrating activation of ERK1/2 and AKT in HCT116-Rab11-FIP4 group (fold change $\geq 2.0$ ). (E) Activation of ERK1/2 and AKT detected by western blot following treatment with IGF1R inhibitor (NVP-AEW541; $5 \mu \mathrm{M}$ ) for $12 \mathrm{~h}$. (F) Migration assay and (G) invasion assay performed using HCT116-Rab11-FIP4 cells treated with either ERK inhibitor (U0126; $5 \mu \mathrm{M})$ or AKT inhibitor (LY294002; $10 \mu \mathrm{M})$ for $6 \mathrm{~h}$, respectively. ${ }^{*} \mathrm{P}<0.05$; ${ }^{* *} \mathrm{P}<0.01$. CRC, colorectal cancer; Rab11-FIP4, Rab11-family interacting protein 4; ERK1/2, extracellular signal-regulated kinase 1/2; AKT, AKT serine/threonine kinase; IGF1R, insulin-like growth factor receptor 1; Co-IP, co-immunoprecipitation.

samples was also analyzed. Based on analysis of integrated optical density value, it was revealed that there was a significant positive correlation between expression of Rab11-FIP4 and HIF-1 $\alpha$ (Fig. 4B). In addition, whether hypoxia could induce Rab11-FIP4 expression in CRC was investigated. HCT116 and LoVo cells were exposed to hypoxic conditions for up to $48 \mathrm{~h}$, and the expression level of Rab11-FIP4 was then analyzed. The results demonstrated that mRNA and protein levels of Rab11-FIP4 were significantly increased under hypoxic conditions (Fig. 4C and D). Furthermore, whether Rab11-FIP4 is a transcriptional target of HIF-1 $\alpha$ in $\mathrm{CRC}$ cells was investigated. There were four putative hormone response elements (HREs) located at the transcriptional start site of Rab11FIP4. Using the dual-luciferase reporter assay, it was revealed that the deletion of the HRE2 site significantly decreased the promoter activity of Rab11-FIP4, which was induced by HIF-1 $\alpha$ (Fig. 4E). These results demonstrate that Rab11-FIP4 is a target gene of HIF-1 $\alpha$ in CRC. 
A

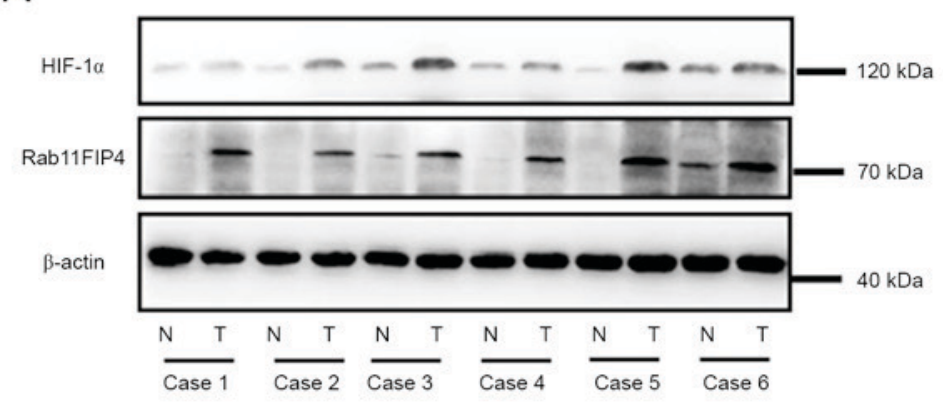

B

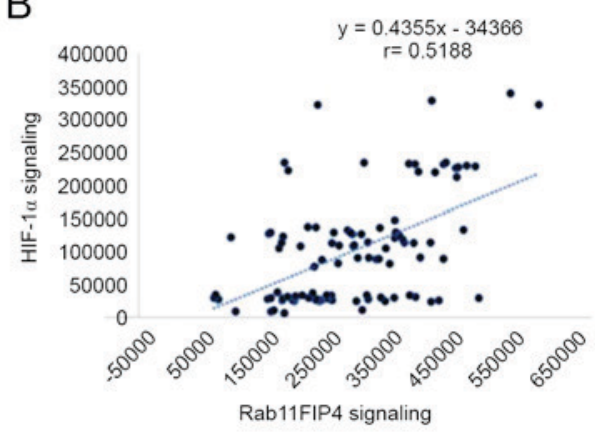

C

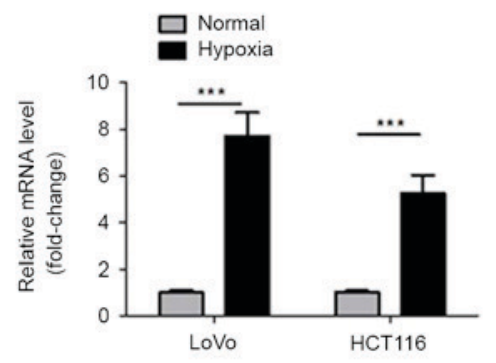

E

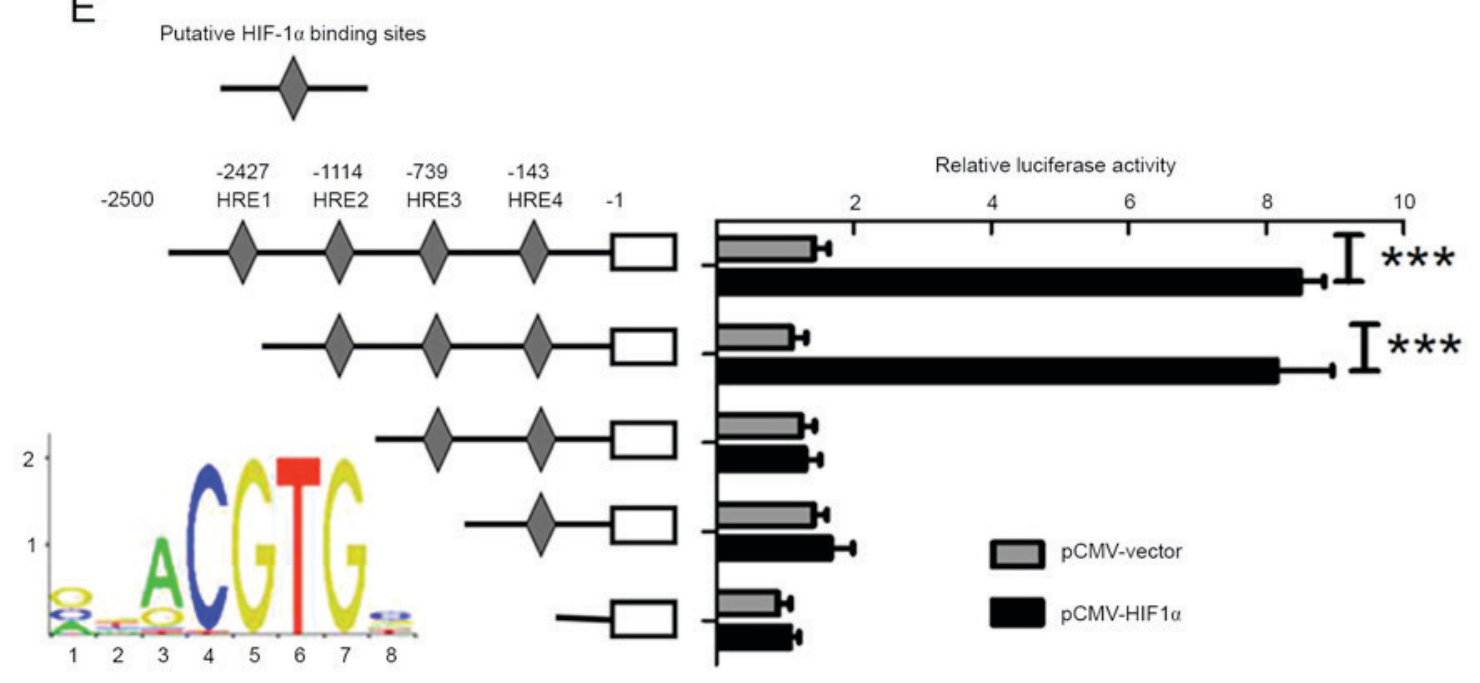

D

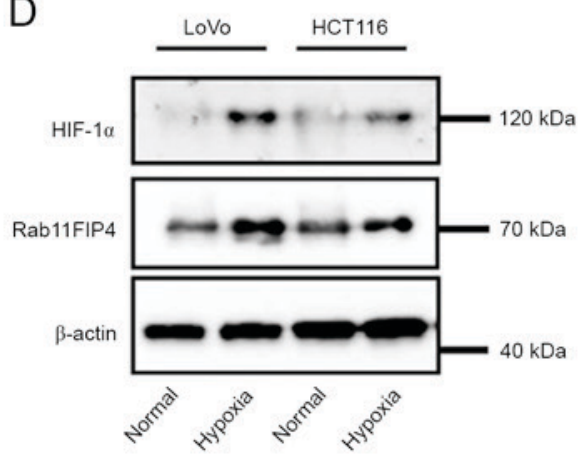

Figure 4. HIF-1 $\alpha$ induces the expression of Rab11-FIP4 in CRC cells. (A) Western blot analysis revealing the expression of Rab11-FIP4 and HIF-1 $\alpha$ levels in six pairs of CRC tissues and corresponding NT tissues. (B) Tissue microarrays of 100 patients with CRC demonstrating the correlation between Rab11-FIP4 and HIF-1 $\alpha$. (C) Reverse transcription-quantitative polymerase chain reaction and (D) western blot analysis of Rab11-FIP4 expression levels in CRC cells exposed to hypoxic conditions for up to $48 \mathrm{~h}$. (E) Dual-luciferase reporter assay results presenting identification of HREs and activity in the Rab11-FIP4 promoter. Gray diamonds represent the four putative HREs. Constructs of truncated Rab11-FIP4 promoter were co-transfected with pCMV6-HIF-1 $\alpha$ or pCMV6 and pRL-TK in HCT116 cells. Normalization was to Renilla luciferase activity. Relative luciferase activity was exhibited as comparison with the pGL3-Basic. ${ }_{* * *}^{*} \mathrm{P}<0.001$. CRC, colorectal cancer; Rab11-FIP4, Rab11-family interacting protein 4; HIF-1 $\alpha$, hypoxia-inducible factor-1 $\alpha$; NT, non-tumoral; HRE, hormone response element.

\section{Discussion}

Cancer invasion and distant metastasis, likely driven by upregulation of oncogene activity or loss of tumor suppressors, are the leading causes of cancer-associated mortality in the majority of cancers, including CRC (4). Due to metastasis, patients with CRC who undergo surgical resection or chemotherapy still face poor survival rates (5). Thus far, the key factors responsible for metastasis in patients with cancer have not yet been determined. In the present study, it was demonstrated that the expression levels of Rab11-FIP4 were significantly higher in CRC tissues compared with corresponding NT tissues, and were associated with overall survival and time until recurrence of patients with CRC. High expression levels of Rab11-FIP4 promoted proliferation, invasion and metastasis of CRC cells. To the best of our knowledge, this study revealed for the first time that Rab11-FIP4 may be an oncogene implicated in CRC.

Rab11 small G proteins (Rab11a, Rab11b and Rab25), members of the Ras superfamily, share high sequence identity and are regulators of the surface expression of receptors and 
adhesion proteins $(11,20)$. Numerous studies have suggested that Rab11 may regulate the transport of several receptors and adhesion proteins, including the $\alpha$-amino-3-hydroxy-5-meth yl-4-isoxazolepropionic acid receptor, rhodopsin, epidermal growth factor receptor, Toll-like receptor $4, \alpha 5 \beta 1$ integrin, E-cadherin and N-cadherin (21). Rab11 directly interacts with the myosin $\mathrm{Vb}$ (MyoVb) globular tail domain, while the C-terminal of MyoVb interacts with the Rab11-FIPs. Furthermore, numerous studies have revealed that members of Rab11-FIPs interact with Rab11. Using a high throughput yeast two-hybrid screen, Fukuda et al (22) demonstrated an interaction between Rab14 and FIP2. Furthermore, Lall et al (9) suggested that all Rab11-FIPs interact with Rab14 and the class I FIPs (RCP, FIP2 and Rip11), but not the class II FIPs (FIP3 and FIP4). In addition, other studies have demonstrated that FIP2 and FIP3 RBDs in complex with Rab11 form a heterotetrameric structure with dyad symmetry (23-25). In the study, it was revealed that increased expression of Rab11-FIP4 significantly increased the protein level of IGF1R, but had no effect on the level of IGF1R mRNA. To the best of our knowledge, this study is the first to demonstrate that Rab11-FIP4 may form a complex with Rab11 and IGF1R, and that increased expression of Rab11-FIP4 increases the formation of this complex in HCT116 cells. However, the exact structural, biophysical and cellular mechanisms underlying this process have not yet been determined.

Molecular and clinical evidence have suggested that the insulin-like growth factor (IGF)/IGF1R system, including IGF, IGF1R and IGF binding proteins, is implicated the in proliferation, differentiation, migration, invasion and angiogenesis of solid cancer cells (26). IGF1R is commonly overexpressed and activated in CRC tissues, and participates in the progression and metastasis of CRC (27). By binding of its ligands, IGF1 or IGF2, the intrinsic tyrosine kinase activity of IGF1R is activated, resulting in its autophosphorylation, and subsequent activation of AKT and mitogen-activated protein kinase pathways $(28,29)$. Furthermore, analysis of human phosphokinase array assay results and the effects of IGF1R inhibitor treatment demonstrated that levels of p-ERK1/2 and p-AKT were significantly increased following overexpression of Rab11-FIP4 in HCT116 cells, however, this was reversed following administration of an IGF1R inhibitor. In addition, CRC cells treated with either ERK1/2 or AKT inhibitors exhibited significantly reduced levels of migration and invasion. These results further suggest that ERK1/2 and AKT signaling are implicated in the tumor promoting function of Rab11-FIP4.

Rapid growth of solid tumors creates a hypoxic microenvironment, which can promote the angiogenesis and metastasis of a tumor. HIF-1 $\alpha$ is stabilized by a hypoxic microenvironment, and induces the expression of a series of target genes involved in CRC metastasis (30). A recent study demonstrated that Rab11-FIP4 was a direct target gene of HIF-1 $\alpha$ in HCC (17). In this study, it was also revealed that there was a significant positive correlation between expression levels of Rab11-FIP4 and HIF-1 $\alpha$ in CRC tissues. Under hypoxic conditions, levels of Rab11-FIP4 and HIF-1 $\alpha$ were significantly increased. The present study also demonstrated that HIF-1 $\alpha$ could induce Rab11-FIP4 transcription by directly binding to its HRE site in the promoter. In conclusion, the results suggest that Rab11-FIP4 is a target gene of HIF-1 $\alpha$. Hypoxia and hypoxia-mediated signaling has a critical role in solid tumor progression. The present study provided evidence for a novel mechanism of hypoxia-mediated oncogenic signaling in CRC progression and Rab11-FIP4 may be a potential target in therapies for the prevention and treatment of CRC.

\section{Acknowledgements}

The present study was supported by grants from the Zhejiang Provincial Natural Science Foundation of China (grant no. LY16H160055) and the Wenzhou Science and Technology Bureau (grant no. Y20150156).

\section{References}

1. Torre LA, Bray F, Siegel RL, Ferlay J, Lortet-Tieulent J and Jemal A: Global cancer statistics, 2012. CA Cancer J Clin 65: 87-108, 2015.

2. Wakamura K, Kudo Se, Miyachi H, Hayashi S, Maeda Y, Kouyama Y, Ichimasa K, Toyoshima N, Misawa M, Mori Y, et al: Mo1695 the prognosis of colorectal cancer patients with negative fecal immunochemical tests: The long-term follow-up study. Gastroenterology 150 (Suppl 1): S754-S755, 2016.

3. Chen W, Zheng R, Baade PD, Zhang S, Zeng H, Bray F, Jemal A, Yu XQ and He J: Cancer statistics in China, 2015. CA Cancer J Clin 66: 115-132, 2016.

4. Lai Y, Wang C, Civan JM, Palazzo JP, Ye Z, Hyslop T, Lin J, Myers RE,Li B, Jiang B, et al: Effects of cancer stage and treatment differences on racial disparities in survival from colon cancer: A united states population-based study. Gastroenterology 150: 1135-1146, 2016.

5. Vatandoust S, Price TJ and Karapetis CS: Colorectal cancer: Metastases to a single organ. World J Gastroenterol 21: 11767-11776, 2015.

6. Prekeris R, Klumperman J and Scheller RH: A Rab11/Rip11 protein complex regulates apical membrane trafficking via recycling endosomes. Mol Cell 6: 1437-1448, 2000.

7. Lindsay AJ, Hendrick AG, Cantalupo G, Senic-Matuglia F, Goud B, Bucci C and McCaffrey MW: Rab coupling protein (RCP), a novel Rab4 and Rab11 effector protein. J Biol Chem 277: 12190-12199, 2002.

8. Wallace DM, Lindsay AJ, Hendrick AG and McCaffrey MW: The novel Rab11-FIP/Rip/RCP family of proteins displays extensive homo- and hetero-interacting abilities. Biochem Biophys Res Commun 292: 909-915, 2002.

9. Lall P, Lindsay AJ, Hanscom S, Kecman T, Taglauer ES, McVeigh UM, Franklin E, McCaffrey MW and Khan AR: Structure-function analyses of the interactions between Rab11 and Rab14 small GTPases with their shared effector rab coupling protein (RCP). J Biol Chem 290: 18817-18832, 2015.

10. Nagase T, Kikuno R and Ohara O: Prediction of the coding sequences of unidentified human genes. XXII. The complete sequences of 50 new cDNA clones which code for large proteins. DNA Res 8: 319-327, 2001.

11. Welz T, Wellbourne-Wood J and Kerkhoff E: Orchestration of cell surface proteins by Rab11. Trends Cell Biol 24: 407-415, 2014.

12. Horgan CP and McCaffrey MW: The dynamic Rab11-FIPs. Biochem Soc Trans 37: 1032-1036, 2009.

13. Meyers JM and Prekeris R: Formation of mutually exclusive Rab11 complexes with members of the family of Rab11-interacting proteins regulates Rab11 endocytic targeting and function. J Biol Chem 277: 49003-49010, 2002.

14. Wallace DM, Lindsay AJ, Hendrick AG and McCaffrey MW: Rab11-FIP4 interacts with Rab11 in a GTP-dependent manner and its overexpression condenses the Rab11 positive compartment in HeLa cells. Biochem Biophys Res Commun 299: 770-779, 2002.

15. Muto A, Arai K and Watanabe S: Rab11-FIP4 is predominantly expressed in neural tissues and involved in proliferation as well as in differentiation during zebrafish retinal development. Dev Biol 292: 90-102, 2006.

16. Muto A, Aoki Y and Watanabe S: Mouse Rab11-FIP4 regulates proliferation and differentiation of retinal progenitors in a Rab11-independent manner. Dev Dyn 236: 214-225, 2007. 
17. Hu F, Deng X, Yang X, Jin H, Gu D, Lv X, Wang C, Zhang Y, Huo X, Shen Q, et al: Hypoxia upregulates Rab11-family interacting protein 4 through HIF-1 $\alpha$ to promote the metastasis of hepatocellular carcinoma. Oncogene 34: 6007-6017, 2015.

18. Xu CL, Wang JZ, Xia XP, Pan CW, Shao XX, Xia SL, Yang SX and Zheng B: Rab11-FIP2 promotes colorectal cancer migration and invasion by regulating PI3K/AKT/MMP7 signaling pathway. Biochem Biophys Res Commun 470: 397-404, 2016.

19. Livak KJ and Schmittgen TD: Analysis of relative gene expression data using real-time quantitative PCR and the 2(-Delta Delta C(T)) method. Methods 25: 402-408, 2001.

20. Prekeris R: Rabs, Rips, FIPs, and endocytic membrane traffic. Scientific World Journal 3: 870-880, 2003.

21. Kelly EE, Horgan CP and McCaffrey MW: Rab11 proteins in health and disease. Biochem Soc Trans 40: 1360-1367, 2012.

22. Fukuda M, Kanno E, Ishibashi K and Itoh T: Large scale screening for novel rab effectors reveals unexpected broad Rab binding specificity. Mol Cell Proteomics 7: 1031-1042, 2008.

23. Eathiraj S, Mishra A, Prekeris R and Lambright DG: Structural basis for Rab11-mediated recruitment of FIP3 to recycling endosomes. J Mol Biol 364: 121-135, 2006.

24. Jagoe WN, Lindsay AJ, Read RJ, McCoy AJ, McCaffrey MW and Khan AR: Crystal structure of rab11 in complex with rab11 family interacting protein 2. Structure 14: 1273-1283, 2006.
25. Shiba T, Koga H, Shin HW, Kawasaki M, Kato R, Nakayama K and Wakatsuki S: Structural basis for Rab11-dependent membrane recruitment of a family of Rab11-interacting protein 3 (FIP3)/Arfophilin-1. Proc Natl Acad Sci USA 103: 15416-15421, 2006.

26. Weroha SJ and Haluska P: The insulin-like growth factor system in cancer. Endocrinol Metab Clin North Am 41: 335-350, 2012.

27. Shali H, Ahmadi M, Kafil HS, Dorosti A and Yousefi M: IGF1R and c-met as therapeutic targets for colorectal cancer. Biomed Pharmacother 82: 528-536, 2016.

28. Martinez-Quetglas I, Pinyol R, Dauch D, Torrecilla S, Tovar V, Moeini A, Alsinet C, Portela A, Rodriguez-Carunchio L, Solé M, et al: IGF2 is up-regulated by epigenetic mechanisms in hepatocellular carcinomas and is an actionable oncogene product in experimental models. Gastroenterology 151: 1192-1205, 2016.

29. Jin H, Wang C, Jin G, Ruan H, Gu D, Wei L, Wang H, Wang N, Arunachalam E, Zhang Y, et al: Regulator of calcineurin 1 gene isoform 4, down-regulated in hepatocellular carcinoma, prevents proliferation, migration, and invasive activity of cancer cells and metastasis of orthotopic tumors by inhibiting nuclear translocation of NFAT1. Gastroenterology 153: 799-811.e33, 2017.

30. Nagaraju GP, Bramhachari PV, Raghu G and El-Rayes BF: Hypoxia inducible factor-1 $\alpha$ : Its role in colorectal carcinogenesis and metastasis. Cancer Lett 366: 11-18, 2015. 